\title{
Dinâmica do Carbono no Solo em Ecossistemas Nativos e Plantações Florestais em Santa Catarina
}

\author{
Silmar Primieri ${ }^{1}$, Aleksander Westphal Muniz ${ }^{2}$, Henrique de Melo Lisboa ${ }^{3}$ \\ ${ }^{1}$ Instituto Federal de Santa Catarina - IFSC, Lages/SC, Brasil \\ ${ }^{2}$ Empresa Brasileira de Pesquisa Agropecuária Amazônia Ocidental - EMBRAPA, Manaus/AM, Brasil \\ ${ }^{3}$ Universidade Federal de Santa Catarina - UFSC, Florianópolis/SC, Brasil
}

\section{RESUMO}

A mudança no uso da terra, aliada às práticas de manejo, contribui para as alterações entre as entradas e saídas de carbono do solo. O objetivo deste trabalho foi avaliar a dinâmica do carbono no solo em ecossistemas nativos e plantações florestais de Santa Catarina. O trabalho foi conduzido entre os meses de fevereiro e maio de 2007. As áreas avaliadas foram: Campo Nativo (CN); Floresta Ombrófila Mista (FOM); Reflorestamento de Eucalipto (RE) e Pinus (RP). Foram analisados os estoques de carbono, carbono na biomassa microbiana (CBM); respiração basal do solo (RBS) e quocientes microbiano e metabólico ( $\mathrm{qMic}$ e $\mathrm{qCO}_{2}$ ). As maiores concentrações de carbono foram encontradas na FOM e no $\mathrm{CN}$, e os atributos bioquímicos indicam que o $\mathrm{CN}$ e a FOM possuem a melhor capacidade de armazenar carbono no solo, entre todos os sistemas de uso da terra analisados. Nos reflorestamentos de Eucalipto e Pinus, os altos índices de RBS, $\mathrm{qCO}_{2} \mathrm{e}$ as baixas concentrações de CBM sugerem que esses sistemas de uso da terra perdem mais $\mathrm{CO}_{2}$ para atmosfera do que os ambientes nativos.

Palavras-chave: estoque de carbono, biomassa microbiana, mudança no uso da terra.

\section{Soil Carbon Dynamics in Native Ecosystems and Reforestation in Santa Catarina}

\begin{abstract}
Changes in land use combined with management practices contribute to changes in soil carbon inputs and outputs. This study aimed to evaluate the soil carbon dynamics in native ecosystems and reforestation in Santa Catarina. The study was conducted between February and May, 2007. The following areas were evaluated: Native Field (NF); Araucaria Forest (AF); Eucalyptus (ER) and Pinus (PR) reforestation. Carbon stocks, carbon microbial biomass (CMB), soil basal respiration (SBR), microbial (qMic) and metabolic (qCO2) quotients were analyzed. The highest concentrations of carbon were found in $\mathrm{AF}$ and $\mathrm{NF}$, and biochemical characteristics indicated that $\mathrm{NF}$ and AR have the best capacity to store carbon in soil among the land use systems evaluated. Eucalyptus and Pinus reforestation showed high rates of SBR, $\mathrm{qCO} 2$ and low concentrations of $\mathrm{CMB}$ that suggest these land use systems lose more CO2 to atmosphere than native ecosystems.
\end{abstract}

Keywords: carbon stocks, microbial biomass, land use change. 


\section{INTRODUÇÃO E OBJETIVOS}

Os solos contêm cerca de duas vezes mais carbono que a atmosfera (IPCC, 2007). Portanto, o equilíbrio entre as entradas e saídas de carbono orgânico do solo (COS) tem uma crítica influência sobre a concentração de $\mathrm{CO}_{2}$ atmosférico. As mudanças no uso da terra são as atividades humanas de maior impacto sobre este equilíbrio. Lal (2006) reporta que a magnitude das perdas de carbono pode chegar de 25 a $75 \%$, dependendo dos níveis anteriores de uso da terra, manejo e clima. Melhor compreensão dos impactos do uso da terra no balanço de carbono terrestre do mundo é, portanto, uma condição necessária como parte do esforço global para mitigar a mudança climática.

O plantio de florestas tem demonstrado grande potencial para o sequestro de carbono no solo, uma vez que trata de um manejo de longo prazo, em que os teores de matéria orgânica tendem a estabilizar-se em um novo equilíbrio, acumulando diversas vantagens, como: aumento da biodiversidade, favorecimento da fauna do solo, aporte de matéria orgânica, menores perdas de solo, carbono orgânico e nutrientes pela erosão e aumento da capacidade de retenção de água no solo (Campanha et al., 2009). No entanto, o fluxo de nutrientes pode sofrer importantes alterações, com impacto negativo sob o funcionamento da biomassa microbiana do solo (Kaschuk et al., 2011). De modo geral, as áreas cultivadas possuem baixos níveis de saturação de carbono, em torno de 50\% (Wiesmeier et al., 2014), e poderiam estocar quantidades consideráveis do carbono da atmosfera.

O solo comporta-se como um sistema aberto, trocando matéria e energia com o meio. As alterações no estoque de carbono no solo são controladas pela taxa de decomposição de COS, por exemplo, devido a alterações no microclima e pelas alterações na quantidade e na qualidade do $\mathrm{C}$ que circula através do sistema. O uso da terra afeta diretamente tanto microclima quanto a quantidade, a qualidade e as vias de entrada C. Além disso, a erosão é controlada pelo uso e manejo da terra e pode diminuir os estoques de COS em sistemas agrícolas em comparação com as florestas (Don et al., 2011). Dessa forma, a quantidade de carbono adicionada depende do tipo de cultura e das espécies utilizadas. Já as perdas de carbono ocorrem principalmente pela liberação de $\mathrm{CO}_{2}$ na respiração, pela decomposição microbiana dos resíduos e da matéria orgânica do solo e pelas perdas de compostos orgânicos por lixiviação e erosão. A magnitude desses processos depende direta ou indiretamente do manejo do solo (Mielniczuk, 1999).

O solo possui intensa atividade biológica e contém populações microbianas balanceadas, sendo vários os indicadores microbiológicos que podem ser utilizados para estimar sua qualidade. Por isso, o conhecimento dos fatores que afetam os microrganismos do solo é fundamental para a manutenção sustentável do ambiente (Tótola \& Chaer, 2002).

O objetivo deste trabalho foi avaliar a dinâmica do carbono no solo em ecossistemas nativos e plantações florestais no Planalto Serrano de Santa Catarina.

\section{MATERIAL E MÉTODOS}

O trabalho foi realizado no município de Campo Belo do Sul, em Santa Catarina (SC), na área da empresa Florestal Gateados LTDA, às margens da Rodovia SC-459, km 159. O clima na região é do tipo subtropical úmido, classificado parcialmente como $\mathrm{Cfb}$ e Cfa, de acordo com o método de Köppen. O relevo da área é do tipo planalto, com altitudes que variam de 700 a 1.050 metros acima do nível do mar. O solo foi caracterizado através de tradagens, observação em cortes de estradas e avaliação da cor e textura, como associação de Nitossolo Háplico e Cambissolo Húmico, com inclusões de Neossolos Litólicos.

A temperatura média mensal e a precipitação mensal foram medidas pela Estação Meteorológica da Epagri de Lages/SC durante os meses de fevereiro a maio de 2007 e comparadas com a média histórica de sete anos anteriores. Com isso, pode-se perceber que a temperatura da atmosfera na época de coleta seguiu a tendência média para a região. No entanto, os dados pluviométricos mostraram variação em torno da média esperada, com períodos de menor e maior disponibilidade hídrica nos meses de coleta (Figura 1).

As amostras foram coletadas mensalmente de fevereiro a maio de 2007, em quatro diferentes tipos de cobertura vegetal: Floresta Ombrófila Mista (FOM); Campo Nativo (CN) sem pastejo desde 1990 e roçado a cada dois anos; Reflorestamento de Pinus taeda (RP) e Reflorestamento de Eucalyptus dunnii (RE), ambos com 12 anos de idade. 
As amostras foram determinadas de forma aleatória dentro de cada tipo de cobertura vegetal. A coleta do solo aconteceu de forma sistemática, eliminando a serrapilheira sobre a superfície, à profundidade de 0 a $20 \mathrm{~cm}$. Para cada tipo de cobertura vegetal foram determinadas 12 pontos de amostragem de solo, dentro de uma parcela $20 \times 20 \mathrm{~m}$, distantes num raio de, aproximadamente, $5 \mathrm{~m}$. As parcelas foram instaladas em áreas geograficamente próximas, com no máximo $5 \mathrm{~km}$ de distância, assim, diminuindo a interferência de fatores ambientais, como a temperatura e precipitação.

O carbono orgânico total do solo foi estimado pelo método de oxidação da matéria orgânica via úmida, com dicromato de potássio em meio sulfúrico e titulado com solução padrão de sulfato ferroso. A densidade das amostras foi determinada pelo método do anel

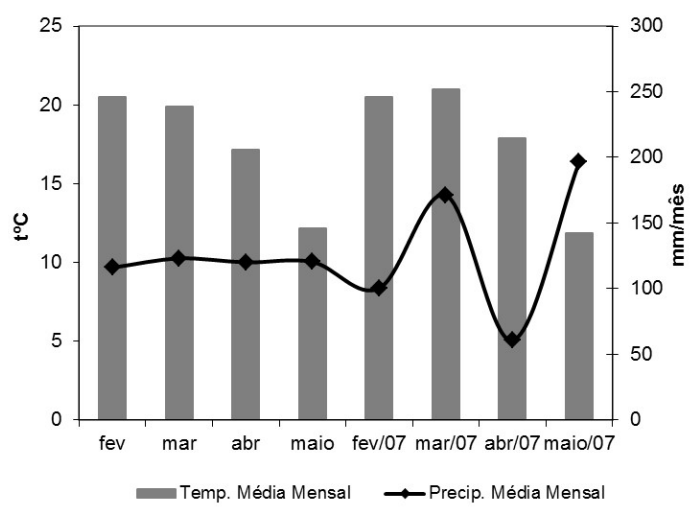

Figura 1. Médias mensais de temperatura e precipitação registradas entre os meses de fevereiro e maio de sete anos anteriores (2000-2006) e durante o período de coleta, em 2007.

Figure 1. Monthly averages of temperature and precipitation recorded between February and May of seven years $(2000-2006)$ and during the sampling in 2007. volumétrico, com três repetições (EMBRAPA, 1997). O cálculo do estoque de carbono no solo $\left(\mathrm{Mg} \mathrm{ha}^{-1}\right)$ foi realizado multiplicando o teor de carbono orgânico do solo, por sua densidade $\left(\mathrm{g} \mathrm{cm}^{3}\right)$, convertida para $\mathrm{Mg}$ e pela profundidade $(\mathrm{cm})$, de acordo com a fórmula abaixo, em que Ct é o estoque de carbono, Cé o teor de carbono orgânico do solo (\%), D é a densidade e P é a profundidade analisada (Szakács, 2003) (Equação 1).

$C t(M g / h a)=C \times D \times P$

A caracterização microbiológica foi feita imediatamente após a coleta, sendo obtida pela análise dos seguintes atributos: carbono da biomassa microbiana (CBM), avaliado pelo método de fumigação-extração, conforme Vance et al. (1987); respiração basal do solo (RBS), de acordo com Jenkinson \& Powlson (1976). O quociente metabólico $\left(\mathrm{qCO}_{2}\right)$ foi determinado pela razão entre a respiração basal do solo por unidade de CBM (Anderson \& Domsch, 1985, 1990) e o quociente microbiano (qMIC), pela razão entre o CBM e o Carbono Orgânico Total (COT) do solo. As análises químicas (Tabela 1) foram realizadas de acordo com Tedesco et al. (1985).

Os dados experimentais foram analisados em design completamente casualisados, com doze repetições, e submetidos à análise de variância (ANOVA). Todos os pressupostos foram avaliados. A normalidade do erro foi avaliada pelo teste de Shapiro Wilk (Shapiro \& Wilk, 1965), a homogeneidade da variância pelo teste de Levene (O’Neill \& Mathews, 2000). Quando as diferenças foram significativas, as médias foram comparadas pelo teste de Tukey ao nível de 5\% de probabilidade, para caracterizar as diferenças entre os tratamentos. A análise estatística foi realizada com auxílio do programa $R$ (R Development Core Team, 2014).

Tabela 1. Características químicas do solo em quatro diferentes usos da terra: Campo Nativo (CN); Floresta Ombrófila Mista (FOM); Reflorestamento de Eucalipto (RE) e Reflorestamento de Pinus (RP).

Table 1. Chemical characteristics of soil in four different land uses: Native Field (NF); Araucaria Forest (AF); Eucalyptus Reforestation (ER) and Pinus reforestation (PR).

\begin{tabular}{|c|c|c|c|c|c|c|c|c|}
\hline \multirow{2}{*}{$\begin{array}{c}\text { Sistema } \\
\text { de Uso da } \\
\text { Terra }\end{array}$} & \multirow[b]{2}{*}{$\operatorname{Arg}^{\star}(\%)$} & \multirow[b]{2}{*}{$\mathrm{pH}$} & $\mathbf{P}$ & $\mathbf{K}$ & Al & $\mathrm{Ca}$ & Mg & CTC \\
\hline & & & \multicolumn{2}{|c|}{---Mg/dm---- } & \multicolumn{4}{|c|}{-----Cmolc/dm------ } \\
\hline $\mathrm{CN}$ & 46,87 & 4,30 & 2,12 & 102,08 & 4,95 & 1,47 & 1,05 & 31,39 \\
\hline FOM & 41,54 & 3,82 & 3,20 & 73,41 & 5,42 & 2,11 & 0,82 & 41,69 \\
\hline $\mathrm{RP}$ & 56,29 & 4,96 & 3,20 & 39,54 & 1,01 & 3,73 & 2,21 & 15,29 \\
\hline $\mathrm{RE}$ & 51,31 & 4,21 & 3,39 & 34,79 & 4,55 & 1,11 & 1,14 & 34,10 \\
\hline
\end{tabular}

*Argila. Média de 12 repetições. 


\section{RESULTADOS E DISCUSSÃO}

\subsection{Estoque de carbono no solo}

Os resultados mostraram maiores taxas de carbono acumulado no solo da Floresta Ombrófila Mista (FOM) em relação aos outros sistemas de uso do solo (Figura 2a). As taxas de carbono no solo do Campo Nativo $(\mathrm{CN})$ foram maiores que no Reflorestamento de Pinus taeda (RP), mas semelhantes aos resultados encontrados no Reflorestamento de Eucalyptus dunnii (RE). Os estoques de carbono no Reflorestamento de Pinus taeda (RP) e no Reflorestamento de Eucalyptus dunnii (RE) não apresentaram diferenças estatísticas.

A menor concentração de carbono em reflorestamentos, quando comparados a Floresta Nativa de Araucária e Campo Nativo, no mesmo tipo de solo, também foi

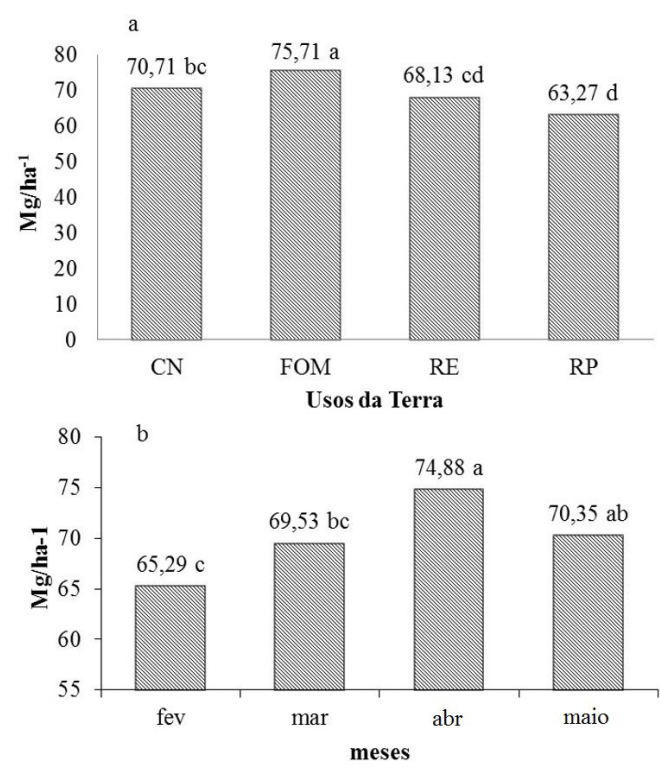

Figura 2. Estoque de carbono (a) em quatro diferentes usos da terra: Campo Nativo (CN); Floresta Ombrófila Mista (FOM); Reflorestamento de Eucalipto (RE) e Reflorestamento de Pinus (RP) e (b) média do estoque de carbono de todos os usos da terra e sua variação entre os meses de fevereiro e maio. Médias seguidas de diferentes letras minúsculas indicam diferenças (ANOVA) a $\mathrm{P} \leq 0,05$.

Figure 2. Carbon stocks (a) in four different land uses: Native Field (NF); Araucaria Forest (AF); Eucalyptus Reforestation (ER) and Pinus reforestation (PR) and (b) carbon stock average of all land use and its variation during the months of February to May. Means followed by different lower case letters indicates differences (ANOVA) at $\mathrm{P} \leq 0.05$. observada por Souza (2004), que analisou o solo na profundidade de $0-10 \mathrm{~cm}$ e encontrou resultados de 47,6 $\mathrm{Mg} \mathrm{ha}^{-1}$ nos reflorestamentos de Pinus com 12 anos de plantio e de 48,4 e 51,7 $\mathrm{Mg} \mathrm{ha}^{-1}$ para o Campo Nativo e para a Floresta Nativa, respectivamente.

Guo \& Gifford (2002) e Guo et al. (2007) encontraram resultados semelhantes, com reduções nos estoques de carbono no solo de até $20 \%$ para plantações de Pinus, comparando com estoques de carbono em Florestas nativas e pastagens naturais na Austrália. Caldeira et al. (2002) demonstraram que a idade do reflorestamento, o tipo de vegetação, entre outros fatores, podem influenciar os estoques de carbono no solo. Em povoamentos com Acacia mearnsii, a maior quantidade de carbono no solo estava nos reflorestamentos mais antigos, enquanto, para povoamentos de Pinus taeda, as concentrações de carbono diminuem nos reflorestamentos acima de 20 anos.

Em monoculturas de Pinus na região sul do Brasil, Trevisan et al. (1987) observaram maior acúmulo de resíduos orgânicos na superfície do solo em relação às florestas nativas, devido à dificuldade de decomposição da fitomassa. O acúmulo de carbono no solo depende da quantidade de material orgânico depositado e de sua taxa de decomposição. Sendo assim, em plantações florestais, espera-se que a biomassa depositada no solo seja variável no tempo, ao contrário de vegetações já estabelecidas, onde a deposição dessa biomassa tende a ser estável.

Essa informação pode ser observada através das alterações nas médias dos estoques de carbono de todos os usos da terra e sua variação durante os meses de fevereiro a maio (Figura 2b), que foi significativa. $\mathrm{O}$ mês de abril apresentou estoques de carbono $(\mathrm{Ct})$ maiores que os meses de fevereiro e março, mas foi semelhante ao mês de maio. Entretanto, os meses de maio e março não diferiram estatisticamente pelo teste de Tukey a $5 \%$.

O comportamento nas concentrações do carbono no solo durante os meses de fevereiro e maio foram diferentes de acordo com o sistema uso da terra (Figura 3). Esse resultado indica a influência do tipo de vegetação sobre a dinâmica do carbono no solo.

Com o maior aporte de matéria orgânica e com as mudanças na temperatura e umidade (Figura 1), ocorrem alterações nas taxas de decomposição (Roscoe et al., 2006). Quando a temperatura diminui 

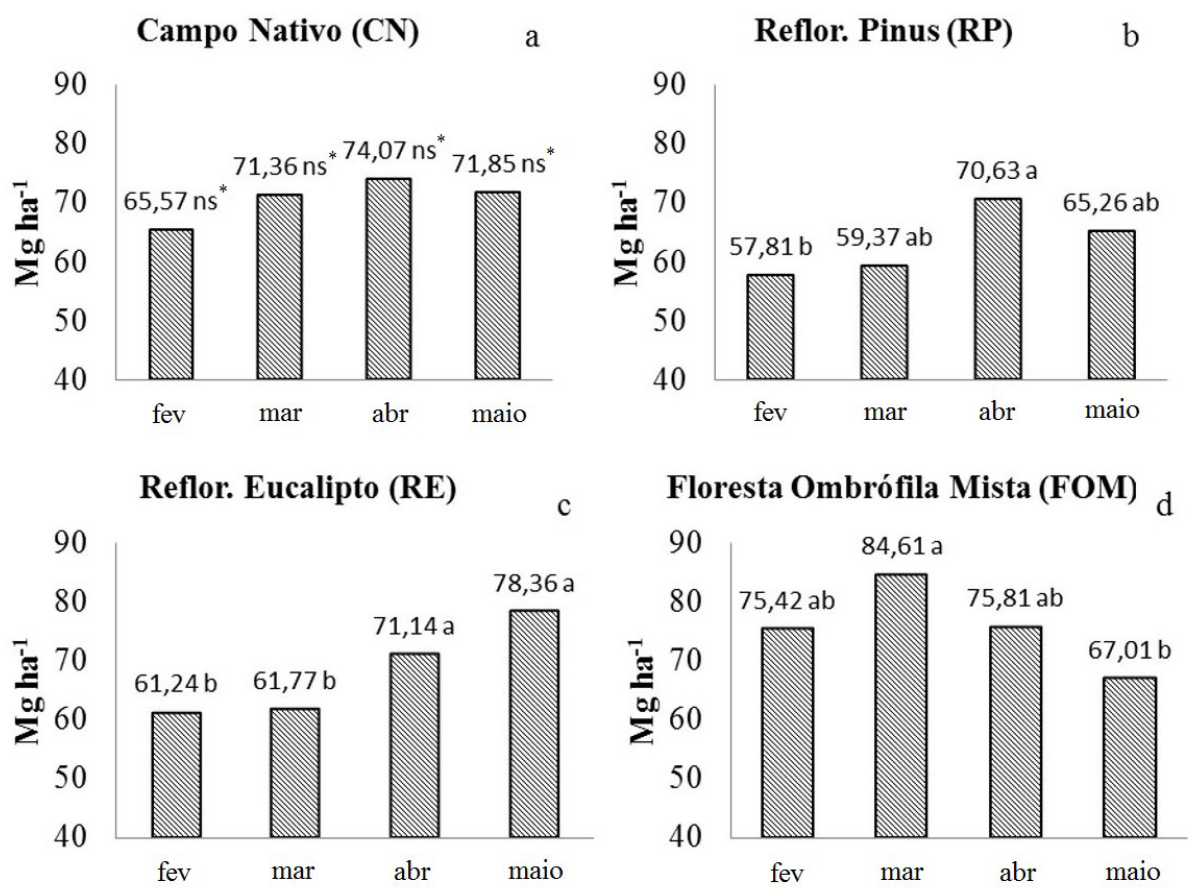

Figura 3. Interações entre os usos da terra e os meses sobre o estoque de carbono no solo (Ct): (a) Campo Nativo (CN); (b) Floresta Ombrófila Mista (FOM); (c) Reflorestamento de Eucalipto (RE) e (d) Reflorestamento de Pinus (RP). Médias seguidas de diferentes letras minúsculas indicam diferenças (ANOVA) a $\mathrm{P} \leq 0,05 .{ }^{*}$ ns: não significativo. Figure 3. Interactions between land uses and months on carbon stocks in soil (Ct): Native Field (NF); Araucaria Forest (AF); Eucalyptus Reforestation (ER) and Pinus reforestation (PR). Means followed by different lower case letters indicates differences (ANOVA) at $\mathrm{P} \leq 0.05$. ${ }^{*}$ ns: not significant.

na região, marca o início da queda de folhas oriunda da vegetação, que é claramente observada na floresta nativa devido à grande diversidade de plantas. Em sistemas homogêneos, como o reflorestamento de Pinus e Eucalipto, ocorre o acréscimo de resíduos vegetais de mesma origem, o que tende a concentrar a disponibilidade desses resíduos em determinada época do ano. A diminuição da temperatura e da umidade do solo influencia negativamente a decomposição da matéria orgânica que é depositada no solo. Devido à lenta decomposição, as concentrações de carbono nos primeiros $20 \mathrm{~cm}$ de profundidade normalmente são maiores (Balbinot et al., 2003).

\subsection{Atividade da biomassa microbiana e da respiração basal do solo}

A atividade da biomassa microbiana do solo reflete o sistema de uso da terra empregado no local e pode aumentar ou diminuir as concentrações de carbono no solo (D’Andréa et al., 2006). Os resultados do Carbono da Biomassa Microbiana (CBM) nos sistemas nativos (CN e FOM) foram maiores em relação aos reflorestamentos Eucalipto (RE) e Pinus (RP), respectivamente, e diferiram estatisticamente (Tabela 2).

A tendência de maiores valores de CBM nos ecossistemas naturais de uso da terra que ambientes alterados pela ação humana também foi encontrada por Matsuoka et al. (2003) e Souza (2004), que reportam a necessidade de trabalhos que estimem o impacto da alteração de uso da terra nos componentes microbiológicos do solo. As camadas superficiais do solo são as mais afetadas pelo uso da terra (1), pois representam grande parte do C que está associado aos microrganismos. Dessa forma, distúrbios na superfície podem afetar negativamente a população microbiana e influenciar a dinâmica do carbono.

A manutenção de sistemas de uso da terra de longo prazo pode auxiliar no aumento da atividade microbiana nas camadas superficiais do solo e refletir 
Tabela 2. Atividade da Biomassa Microbiana do solo em quatro diferentes usos da terra: Campo Nativo (CN); Floresta Ombrófila Mista (FOM); Reflorestamento de Eucalipto (RE) e Reflorestamento de Pinus (RP).

Table 2. Soil Carbon Microbial Biomass activity in four different land uses: Native Field (NF); Araucaria Forest (AF); Eucalyptus Reforestation (ER) and Pinus reforestation (PR).

\begin{tabular}{|c|c|c|}
\hline Sistema de Uso da Terra & $\begin{array}{c}\text { Carbono da Biomassa Microbiana } \\
\text { (CBM) } \\
\left(\mathrm{mg} \mathrm{kg}^{-1} \text { solo }\right)\end{array}$ & $\begin{array}{c}\text { Respiração Basal do Solo (RBS) } \\
\text { mg CO } \mathrm{kg} \mathrm{Solo} \mathrm{h}^{-1}\end{array}$ \\
\hline Campo Nativo (CN) & $1353,60 \mathrm{a}$ & $38,68 \mathrm{bc}$ \\
\hline Floresta Ombrófila Mista (FOM) & $1333,19 \mathrm{a}$ & $36,53 \mathrm{c}$ \\
\hline Reflorestamento de Pinus (RP) & $883,79 \mathrm{~b}$ & $46,60 \mathrm{bc}$ \\
\hline Reflorestamento de Eucalipto (RE) & $799,33 \mathrm{~b}$ & 51,79 a \\
\hline
\end{tabular}

Médias seguidas de diferentes letras minúsculas indicam diferenças (ANOVA) a $\mathrm{P} \leq 0,05$.

no incremento de carbono nessa fração (Anderson \& Domsch, 1985). No entanto, os resultados indicam que mesmo reflorestamentos mantidos por 12 anos ainda possuem quantidades de $\mathrm{C}$ na biomassa microbiana inferiores que os sistemas nativos. Esse resultado pode estar relacionado ao tipo de resíduo vegetal depositado no solo, que nos reflorestamentos tende a ser mais homogêneo, especializando a microbiota.

A tendência de maior estabilidade nos fluxos de carbono do solo também foi observada nos valores da respiração basal do solo (RBS), onde os reflorestamentos de Eucalipto (RE) e Pinus (RP) apresentaram resultados para

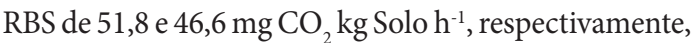
e foram superiores aos valores encontrados no Campo Nativo (CN) e na Floresta Ombrófila Mista (FOM) com 36,7 e 36,5 mg CO $\mathrm{kg} \mathrm{Solo} \mathrm{h}^{-1}$, respectivamente.

Os baixos valores de RBS e altos índices de Carbono na Biomassa Microbiana para os sistemas nativos (FOM e $\mathrm{CN}$ ) significa que esses sistemas perdem menos carbono na forma de $\mathrm{CO}_{2}$, incorporando carbono à biomassa microbiana, sugerindo que essas comunidades estão em estágio mais avançado de sucessão (Carvalho, 2005).

A atividade microbiana pode ser uma característica importante quando se considera que a decomposição dos resíduos orgânicos irá disponibilizar nutrientes para as plantas. No entanto, uma alta RBS pode liberar o carbono contido na matéria orgânica estável e comprometer os processos químicos e físicos do solo, como a agregação de partículas e retenção de água e nutrientes. Portanto, uma alta RBS pode significar tanto um distúrbio como um alto nível de produtividade, dependendo da análise de cada caso (Roscoe et al., 2006).

Essas alterações também são influenciadas pela temperatura e umidade e podem ser observadas na Figura 4. De modo geral, o CBM e a RBS apresentaram
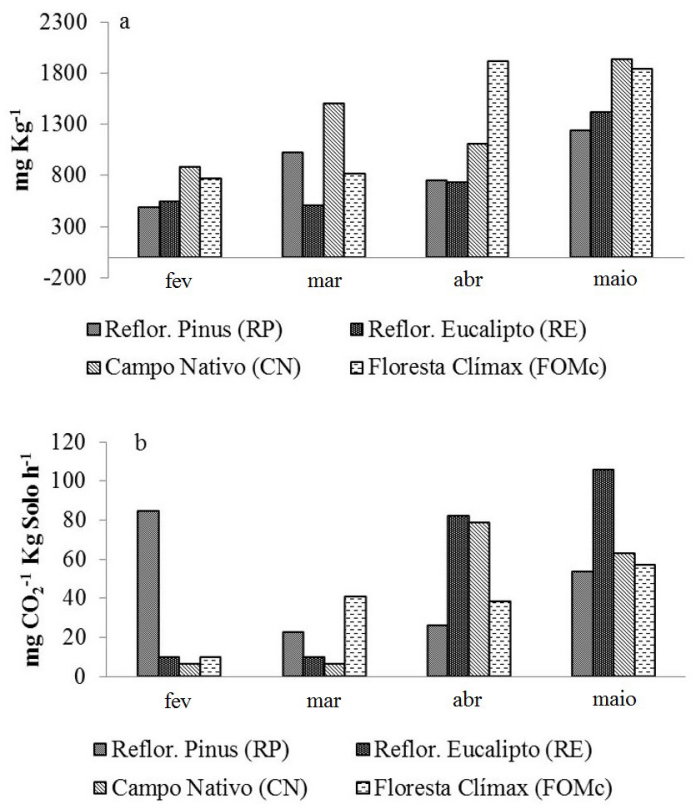

Figura 4. Carbono na biomassa microbiana (a), respiração basal do solo (b) e sua variação entre os meses de fevereiro e maio em quatro tipos de uso da terra: Campo Nativo (CN); Floresta Ombrófila Mista (FOMc); Reflorestamento de Eucalipto (RE) e Reflorestamento de Pinus (RP).

Figure 4. Carbon microbial biomass (a), soil basal respiration (b) and its variation between the months February and May in four different land uses: Native Field (NF); Araucaria Forest (AF); Eucalyptus Reforestation (ER) and Pinus reforestation (PR).

maiores resultados nos meses de abril e maio, que representam o início de temperaturas mais baixas na região. Em florestas de clima temperado, normalmente ocorre um aporte de carbono no solo oriundo da queda das folhas e morte de plantas causadas pela diminuição da temperatura do ambiente durante períodos mais 
Tabela 3. Quociente Microbiano (qMic) e Quociente Metabólico ( $\left.\mathrm{qCO}_{2}\right)$ em quatro diferentes usos da terra: Campo Nativo (CN); Floresta Ombrófila Mista (FOM); Reflorestamento de Eucalipto (RE) e Reflorestamento de Pinus (RP). Table 3. Microbial quotient (qMIC) and Metabolic Quotient (qCO2) in four different land uses: Native Field (NF); Araucaria Forest (AF); Eucalyptus Reforestation (ER) and Pinus reforestation (PR).

\begin{tabular}{lcc}
\multicolumn{1}{c}{ Sistema de Uso da Terra } & Quociente Microbiano (qMic) & $\begin{array}{c}\text { Quociente Metabólico }\left(\mathbf{q} \mathbf{C O}_{2}\right) \\
\%\end{array}$ \\
\hline Campo Nativo (CN) & $\%$ & $0,03 \mathrm{~b}$ \\
Floresta Ombrófila Mista (FOM) & $4,09 \mathrm{a}$ & $0,03 \mathrm{~b}$ \\
Reflorestamento de Pinus (RP) & $4,19 \mathrm{a}$ & $0,08 \mathrm{a}$ \\
Reflorestamento de Eucalipto (RE) & $3,49 \mathrm{~b}$ & $0,07 \mathrm{a}$ \\
\hline
\end{tabular}

Médias seguidas de diferentes letras minúsculas indicam diferenças (ANOVA) a $\mathrm{P} \leq 0,05$.

frios. Assim, grande parte da decomposição desses materiais é realizada pela atividade de microrganismos no solo, o que estimula seu desenvolvimento e reprodução, aumentando a CBM temporariamente. Cattelan \& Vidor (1990) correlacionaram a atividade microbiana com variações ambientais e encontraram taxas de RBS maiores em temperaturas mais amenas e de melhor disponibilidade hídrica. No entanto, variações nos resultados de CBM e RBS, em relação às diferentes épocas do ano, também foram afetadas significativamente em regiões de clima tropical, como no trabalho de Gama-Rodrigues et al. (2005), que avaliou solos do estado do Espírito Santo, Brasil.

De acordo com o trabalho de Vargas et al. (2010), os processos no ecossistemas são fortemente influenciados pelas perturbações climáticas. Eles mostraram que a temperatura e a umidade do solo influenciam diferentemente a produção de $\mathrm{CO}_{2}$ em múltiplas escalas (horas, dias, semanas, meses e anos).

A atividade microbiana do solo é regulada, entre outros fatores, pela qualidade do material orgânico que constitui a serrapilheira (Mielniczuk, 1999). As taxas de decomposição das acículas do Pinus e folhas de Eucalipto podem interferir na dinâmica metabólica do solo, acumulando substâncias antimicrobianas, o que diminui a atividade e diversidade dos microrganismos. Essa alteração na microbiota do solo interfere diretamente os efluxos de $\mathrm{CO}_{2}$.

\subsection{Quociente microbiano e quociente metabólico}

Os resultados encontrados do Quociente Microbiano (qMic) para os sistemas de uso da terra nativos foram maiores que os valores dos reflorestamentos. Para o Quociente Metabólico ( $\mathrm{qCO}_{2}$ ), o reflorestamento de
Pinus e de Eucalipto apresentaram resultados maiores que o Campo Nativo e a Floresta (Tabela 3).

Esses dois parâmetros (Quociente Microbiano e Quociente Metabólico) são indicadores da atividade microbiológica do solo e auxiliam a identificar se o solo está aumentando ou não seu estoque de carbono. Os resultados de $\mathrm{qCO}_{2}$ apontam que os sistemas de uso da terra com reflorestamentos estão desequilibrados e perdem muito carbono para a atmosfera. Embora os valores de qMic sejam maiores no $\mathrm{CN}$ e na FOM, os menores valores de $\mathrm{qCO}_{2}$ indicam que esses sistemas de uso da terra, com menor interferência humana, apresentam menor perda de $\mathrm{CO}_{2}$ para a atmosfera. Portanto, sua biomassa é mais "eficiente" na ciclagem de nutrientes e armazenamento de carbono. Isto está de acordo com o encontrado por Baretta et al. (2005), que afirmam que o Campo Nativo e a Floresta Ombrófila Mista em estágio clímax são sistemas mais conservativos, com menores perdas de carbono e de maior sustentabilidade.

Moreira \& Siqueira (2002), analisando diversos artigos sobre a atividade metabólica do solo, concluíram que a população microbiana de solos de monoculturas tem necessidade energética mais elevada para sua manutenção, ao contrário do observado em solos sob rotação de culturas e vegetação nativa.

\section{CONCLUSÕES}

Nos reflorestamentos de Eucaliptus e Pinus, os altos índices de $\mathrm{RBS}$ e $\mathrm{qCO}_{2}$ e as baixas concentrações de CBM, em relação ao Campo Nativo e à Floresta Ombrófila Mista, sugerem que esses sistemas de uso da terra podem alterar a dinâmica do carbono no solo, emitindo maior quantidade de $\mathrm{CO}_{2}$ para a atmosfera, pela respiração microbiana. Essa interferência diminui 
os estoques de carbono no solo, aumentando as emissões de $\mathrm{CO}_{2}$ para a atmosfera.

Os resultados mostraram variação entre os meses de fevereiro e maio, dependendo do sistema de uso da terra estabelecido acima do solo, o que reflete a interação entre esses usos e a função desempenhada pela microbiota do solo.

\section{AGRADECIMENTOS}

À Fundação de Amparo à Pesquisa e Inovação do Estado de Santa Catarina - FAPESC pelo apoio financeiro. À Empresa Florestal Gateados pelo apoio logístico e auxílio na condução do experimento e à Empresa de Pesquisa Agropecuária e Extensão Rural de Santa Catarina - EPAGRI pela parceria nas análises dos dados.

\section{STATUS DA SUBMISSÃO}

Recebido: 22 ago., 2014

Aceito: 2 jul., 2016

\section{AUTOR(ES) PARA CORRESPONDÊNCIA}

\section{Silmar Primieri}

Departamento de Biotecnologia, Instituto Federal de Santa Catarina - IFSC, Rua Heitor Villa Lobos, 222, Bairro São Francisco, CEP 88506-400, Lages, SC, Brasil

e-mail: silmar.primieri@gmail.com

\section{REFERENNCIAS}

Anderson JP, Domsch KH. Determination of ecophysiological maintenance carbon requirements of soil microorganisms in a dormant state. Biology and Fertility of Soils 1985; 1(2): 81-89. http://dx.doi.org/10.1007/BF00255134.

Anderson JP, Domsch KH. Application of eco-physiological quociente (qCO2 and $\mathrm{Dq}$ ) on microbial biomasses from soils of different cropping histories. Soil Biology \& Biochemistry 1990; 22(2): 251-255. http://dx.doi. org/10.1016/0038-0717(90)90094-G.

Balbinot R, Schumacker MV, Watzlawick LF, Sanquetta CR. Inventário do carbono orgânico em um plantio de Pinus taeda aos 5 anos de idade no Rio Grande do Sul. Revista Ciências Exatas e Naturais 2003; 5(1): 59-68.

Baretta D, Santos JCP, Figueiredo SR, Klauberg-Filho O. Efeito do monocultivo de Pinus e da queima do campo nativo em atributos biológicos do solo no planalto sul Catarinense. Revista Brasileira de Ciência do Solo 2005; 29(5): 715-724. http://dx.doi.org/10.1590/S010006832005000500007 .

Caldeira MVW, Watzlawick LF, Schumacker MV, Balbinot R, Sanquetta CR. Carbono orgânico em solos florestais. In: Sanquetta CR, Watzlawick LF, Balbinot R, Ziliotto MAB, Gomes FS, editores. As florestas e o carbono. Curitiba: Imprensa Universitária da UFPR, 2002.

Campanha MM, Nogueira RS, Oliveira TS, Teixeira AS, Romero RE. Teores e estoques de carbono no solo de sistemas agroflorestais e tradicionais no semiárido brasileiro. Sobral: EMBRAPA; 2009. Circular Técnica n. 42.

Carvalho F. Atributos bioquímicos como indicadores da qualidade do solo em florestas de Araucaria angustifólia (Bert.) O. Ktze. no estado de São Paulo [dissertação]. São Paulo: Escola Superior de Agricultura Luiz de Queiroz; 2005.

Cattelan AJ, Vidor C. Flutuações no biomassa, atividade e população microbiana do solo, em função de variações ambientais. Revista Brasileira de Ciência do Solo 1990; 14(2): 133-142.

D’Andréa AF, Silva MLN, Silva CA. Emissões de CO2 do solo: métodos de avaliação e influência do uso da terra. In: Roscoe R, Mercante FM, Salton JC, compiladores. Dinâmica da matéria orgânica do solo em sistemas conservacionistas. Dourados: Embrapa; 2006.

Don A, Schumacher J, Freibauer A. Impact of tropical land-use change on soil organic carbon stocks-a metaanalysis. Global Change Biology 2011; 17(4): 1658-1670. http://dx.doi.org/10.1111/j.1365-2486.2010.02336.x.

EMBRAPA. Manual de métodos de análise de solo. Rio de Janeiro: CNPS; 1997.

Gama-Rodrigues EF, Barros NF, Rodrigues ACG, Santos GA. Nitrogênio, carbono e atividade da biomassa microbiana do solo em plantações de eucalipto. Revista Brasileira de Ciência do Solo 2005; 29(6): 893-901.

Guo LB, Cowie AL, Montagu KD, Gifford RM. Carbon and nitrogen stocks in a native pasture and an adjacent 16-year-old Pinus radiata D. Don. plantation in Australia. Agriculture, Ecosystems \& Environment 2007; 124(4): 1-14.

Guo LB, Gifford RM. Soil carbon stocks and land use change: a meta analysis. Global Change Biology 2002; 8(4): 345360. http://dx.doi.org/10.1046/j.1354-1013.2002.00486.x. Intergovernmental Panel Climate Change - IPCC. Climate change 2007: the physical science basis - contribution of Working Group I to the fourth assessment report of the intergovernmental panel on climate change. Paris; 2007 [cited 2013 oct 23]. Available from: http://www.ipcc.ch/ publications_and_data/publications_ipcc_fourth_assessment report_wg1_report_the_physical_science_basis.htm

Jenkinson DS, Powlson DS. The effects of biocidal treatments on metabolism in soil. V. A. method for measuring soil 
biomass. Soil Biology \& Biochemistry 1976; 8(3): 209-213. http://dx.doi.org/10.1016/0038-0717(76)90005-5.

Kaschuk G, Alberton O, Hungria M. Quantifying effects of different agricultural land uses on soil microbial biomass and activity in Brazilian biomes: inferences to improve soil quality. Plant and Soil 2011;338(1-2): 467-481. http:// dx.doi.org/10.1007/s11104-010-0559-z.

Lal R. Soil carbon sequestration in Latin America. In: Lal R, Cerri CC, Bernoux M, Etchevers J, Cerri E, editores. Carbon sequestration in soils of Latin America. New York: Haworth Press; 2006.

Matsuoka M, Mendes IC, Loureiro MF. Biomassa microbiana e atividade enzimática em solos sob vegetação nativa e sistemas agrícolas anuais e perenes na região de Primavera do Leste (MT). Revista Brasileira de Ciência do Solo 2003; 27(3): 425-433. http://dx.doi.org/10.1590/ S0100-06832003000300004.

Mielniczuk J. Matéria orgânica e a sustentabilidade de sistemas agrícolas. In: Santos GA, Camargo FAO, compiladores. Fundamentos da matéria orgânica do solo: ecossistemas tropicais e subtropicais. Porto Alegre: Genesis; 1999.

Moreira FMS, Siqueira JO. Microbiologia e bioquímica do solo. Lavras: Universidade Federal de Lavras; 2002.

O’Neill ME, Mathews K. Theory \& methods: a weighted least squares approach to levene's test of homogeneity of variance. Australian \& New Zealand Journal of Statistics 2000; 42(1): 81-100. http://dx.doi.org/10.1111/1467842X.00109.

R Development Core Team. R: A language and environment for statistical computing. Vienna: R Foundation for Statistical Computing; 2014.

Roscoe R, Boddey RM, Salton JC. Sistemas de manejo e matéria orgânica do solo. In: Roscoe R, Mercante FM, Salton JC, compiladores. Dinâmica da matéria orgânica do solo em sistemas conservacionistas: modelagem matémática e métodos auxiliares. Dourados: EMBRAPA; 2006.
Shapiro SS, Wilk MB. An analysis of variance test for normality. Biometrika 1965; 52(3-4): 591-611. http:// dx.doi.org/10.1093/biomet/52.3-4.591.

Souza IMZ. Carbono e nitrogênio da biomassa microbiana do solo em áreas reflorestadas comparadas ao campo e mata nativa no Planalto dos Campos Gerais, SC [dissertação]. Lages: Programa de Mestrado em Agronomia, Universidade do Estado de Santa Catarina; 2004.

Szakács GGJ. Sequestro de carbono nos solos: avaliação das potencialidades dos solos arenosos sob pastagens, Anhembi/ SP [dissertação]. Piracicaba: Centro de Energia Nuclear na Agricultura, Universidade de São Paulo; 2003.

Tedesco MJ, Gianello C, Bissani CA, Volkweiss SJ. Análises de solo, plantas e outros materiais. Porto Alegre: Universidade Federal do Rio Grande do Sul; 1985. Boletim técnico n. 5.

Trevisan E, Reissmann CB, Koehler CW, Lima JMJC. Morfologia de horizontes orgânicos acumulados sob povoamentos de Pinus taeda L., em três sítios distintos. Revista do Setor de Ciências Agrárias 1987; 9: 59-62.

Tótola MR, Chaer GM. Microrganismos e processos microbiológicos como indicadores de qualidade dos solos. In: Sociedade Brasileira de Ciência do Solo. Tópicos em ciência do solo. Viçosa: UFV; 2002.

Vance ED, Brookes PC, Jenkinson DS. An extraction method for measuring soil microbial biomass C. Soil Biology \& Biochemistry 1987; 19(6): 703-707. http:// dx.doi.org/10.1016/0038-0717(87)90052-6.

Vargas R, Detto M, Baldocchi DD, Allen MF. Multiscale analysis of temporal variability of soil $\mathrm{CO} 2$ production as influenced by weather and vegetation. Global Change Biology 2010; 16(5): 1589-1605. http://dx.doi.org/10.1111/j.13652486.2009.02111.x.

Wiesmeier M, Hübner R, Spörlein P, Geuß U, Hangen E, Reischl A et al. Carbon sequestration potential of soils in southeast Germany derived from stable soil organic carbon saturation. Global Change Biology 2014; 20(2): 653-665. PMid:24038905. http://dx.doi.org/10.1111/gcb.12384. 